Short Communication

\title{
High prevalence of ESBLs in retail chicken meat despite reduced use of antimicrobials in chicken production, France
}

\author{
Tiago Casella $^{\mathrm{a}, \mathrm{b}}$, Mara Correa Lelles Nogueira ${ }^{\mathrm{c}}$, Estelle Saras ${ }^{\mathrm{b}}$, Marisa Haenni ${ }^{\mathrm{b}}{ }^{*}$, \\ Jean-Yves Madec ${ }^{\mathrm{b}}$ \\ a Instituto de Biociências, Letras e Ciências Exatas, Universidade Estadual Paulista "Júlio de Mesquita Filho" (UNESP), São José do Rio Preto, SP, Brazil \\ ${ }^{\mathrm{b}}$ Agence Nationale de Sécurité Sanitaire (ANSES) - Université de Lyon, Unité Antibiorésistance et Virulence Bactériennes, Lyon, France \\ ${ }^{c}$ Faculdade de Medicina de São José do Rio Preto (FAMERP), São José do Rio Preto, SP, Brazil
}

\section{A R T I C L E I N F O}

\section{Keywords:}

Chicken

Meat

ESBL

CTX-M-1

IncI1

\begin{abstract}
A B S T R A C T
Extended-spectrum cephalosporins (ESCs) are critically important antibiotics for humans and their use in animals poses a potential threat for public health. Chicken represents an increasing part of the human diet and has also been regarded as a source of ESC-resistant Enterobacteriaceae because of the worldwide off-label use of ceftiofur, a broad-spectrum cephalosporin. Thus, numerous studies pointed out chicken as a reservoir of ESBL/ pAmpC genes, plasmids and/or clones at risk for humans. In France, late 2011, strong political pressure led to a drastic reduction of ceftiofur use and all other antibiotics in chicken production. Here, we ascertained the potential impact of those efforts on the prevalence of ESC-resistant $E$. coli in retail chicken. From October 2015 to January 2016, of 48 unrelated pieces of meat (chicken legs) belonging to four different brands, 44 (91.7\%) were positive for ESC-resistant $E$. coli. The bla $a_{\text {CтХ-м-1 }}$ gene was highly prevalent $(68 / 74,91.9 \%)$, mostly located on IncI1/ST3 plasmids $(65 / 68,95.6 \%)$. Other ESBL/pAmpC genes (bla $a_{\mathrm{TEM}-52}$, bla $_{\mathrm{SHV}-12}$, bla $_{\mathrm{CMY}-2}$ ) were carried by IncX1, IncI1/ST36, IncI1/ST95, IncA/C or IncK plasmids. The positive isolates were non-clonal, suggesting a horizontal spread of the ESBL/pAmpC genes. Obviously, the strong decrease of antimicrobial use in chicken farms had no impact yet on the ESBL/pAmpC prevalence in retail chicken meat in France. A human source of these ESBL/pAmpC genes is unlikely as bla $_{\mathrm{CTX}-\mathrm{M}-1}$ IncI1/ST3 plasmids are dominant in animals and rare in humans. Our data question the real impact of the decrease of antimicrobial use in chicken production on ESBL contamination of chicken meat and point out the risk of ESBL/AmpCs human transfer through the food chain.
\end{abstract}

\section{Introduction}

Resistance to extended spectrum cephalosporins (ESCs) in Enterobacteriaceae is mostly mediated by Extended-Spectrum BetaLactamases (ESBLs) or plasmidic AmpC (pAmpC), and constitutes a major issue in both human and veterinary medicine. In particular, ESCs have been considered as critically important antibiotics (CIA) in human medicine, and national and international guidelines have also been set up in the veterinary sector in order to prevent inappropriate use of ESCs in animals. Very recently, a Joint Opinion from the European Medicines Agency (EMA) and the European Food Safety Authority (EFSA) was issued on measures to reduce the need to use antimicrobial agents in animal husbandry in the European Union, and the resulting impacts on food safety (EMA, 2017).

After the emergence of ESC-resistant Enterobacteriaceae in foodproducing animals globally in the 2000s, numerous studies have specifically focused their interest on the poultry sector, and revealed the presence of ESBLs/pAmpCs at different levels in the broiler production pyramid, as well as on the surface of chicken carcasses or meat thereof, including at retail (Borjesson et al., 2013; Dierikx et al., 2013). It has been now commonly admitted that the selection of ESBL/pAmpC-producers in chicken has mainly resulted from the worldwide off-label use of ceftiofur - a broad-spectrum cephalosporin of veterinary use - directly administered in ovo (Dutil et al., 2010). Of note, high prevalence rates of ESBL/pAmpC-producing Enterobacteriaceae were also reported in countries with very low antibiotic pressure but that had imported contaminated chicken from countries using ceftiofur (Egervarn et al., 2014). Globally, the focus on chicken is of particular concern since chicken meat represents a substantial proportion of the human diet (14.52 kg per person in 2011 in Europe, according to the Food and Agriculture Organization) (FAO, 2015), which is expected to rise dramatically in the future.

In addition, several studies investigated the possible poultry-tohuman transfer of ESBL/pAmpC producers and suggested chicken as a

\footnotetext{
* Corresponding author

E-mail address: marisa.haenni@anses.fr (M. Haenni).
} 
reservoir of ESBL genes, plasmids and/or clones at risk for humans. Indeed, similar or identical ESC-resistant isolates or ESBL/pAmpC plasmids were found in chicken meat and patients in Denmark and the Netherlands (Huijbers et al., 2014; Kluytmans et al., 2013; Leversteinvan Hall et al., 2011; Overdevest et al., 2011). Even though there has been still no final scientific consensus on this question, the EFSA declared the presence of ESBL-producing $E$. coli in poultry meat a significant hazard to public health (Efsa Panel on Biological Hazards et al., 2012).

In the recent years, there has been a strong scientific and political pressure in Europe in order to decrease the use of antibiotics in veterinary medicine, and especially in food-producing animals. Subsequently, and particularly in France, the off-label use of ceftiofur in the chicken production was drastically reduced. More globally, the total number of tons of all antibiotics (including non CIA) used in this sector also significantly decreased, from 254.41 tons in 2007 to 156.67 tons in 2013 (38.4\% reduction), thus limiting the risk of co-selection of ESCresistant bacteria by the use of non-beta-lactams (ANSES, 2014). As a probable consequence, and thanks to the all-in all-out mode of production together with the very short period of chicken rearing (less than two months), the ESBL/pAmpC prevalence in clinical samples from chicken also decreased remarkably from $22.5 \%$ in 2010 to $2.5 \%$ in 2015 in France (www.resapath.anses.fr). In this context, a reasonably low ESBL/pAmpC prevalence would have been expected in chicken meat as well. Our goal was thus to assess the prevalence of ESC-resistant $E$. coli in chicken meat samples at retail and to characterize molecularly the collected isolates.

\section{Material and methods}

\subsection{Bacterial isolation and identification}

Based on the hypothesis of an ESBL prevalence around $50 \%$ in chicken meat, with a required precision of $10 \%$, a sample size of 97 chicken meat pieces was needed. The main commercial centers from the Lyon, France area were identified and, starting in October 2015, 4 to 6 (depending on availability of products) packed chicken legs from four different commercial brands (A to D) were bought each week. Specific attention was paid to be sure that all pieces presented a different lot number, to have unrelated chicken meat pieces. All pieces of meat had a French origin as stated on the packaging.

Two $10 \mathrm{~g}$ samples of each piece of meat were aseptically partitioned and then submitted to two different protocols in order to maximize the chances of selecting ESBL/pAmpC-producing isolates. For protocol A, $10 \mathrm{~g}$ of meat were incubated overnight in $100 \mathrm{~mL}$ of Brain Heart Infusion (BHI) broth supplemented with cefotaxime $(2 \mathrm{mg} / \mathrm{L})$, and $100 \mu \mathrm{L}$ were then plated on MacConkey agar with cefotaxime $(2 \mathrm{mg} / \mathrm{L})$. For protocol B, $10 \mathrm{~g}$ of meat were incubated overnight in $100 \mathrm{~mL}$ of BHI broth and $100 \mu \mathrm{L}$ were then plated onto selective ChromID ESBL plates (Biomérieux, Marcy l'Etoile, France). One presumptive E. coli of each morphology was collected from both ChromID ESBL and MacConkey plates, and identification was performed using a MALDI-TOF (Brucker).

\subsection{Antibiotic susceptibility testing}

Susceptibility testing was performed by disc diffusion using commercially available discs (MAST, Amiens, France) according to the guidelines of the Antibiogram Committee of the French Society for Microbiology (CA-SFM; www.sfm-microbiologie.org). Susceptibility to 32 beta-lactams and non beta-lactams of veterinary and human interest was tested, and ESBL production was confirmed by the double-disc synergy test. E. coli ATCC 25922 was used as quality control.

\subsection{Beta-lactamase and cephalosporinase genes identification}

The $b a_{\text {CTX-м }}$ genes were detected using a CTX-M group-specific multiplex PCR (Shibata et al., 2006), while the $b l a_{\mathrm{TEM}}, b l a_{\mathrm{SHV}}$ and $b l a_{\mathrm{CMY}-2}$ genes were screened by simplex PCRs (Dallenne et al., 2010). For the CTX-M-1 group, an additional PCR was performed using external primers (ISEcp1L1, 5'-CAGCTTTTATGACTCG; P2D, 5'-CAGCGCTTTTGCCGTCTAAG) to detect the ISEcp1 upstream to the $b l a_{\mathrm{CTX}-\mathrm{M}-1}$ genes. All positive amplicons were sequenced (Genewiz, London, United Kingdom).

\subsection{Transferability of the bla $a_{E S B L / P A m p C}$ genes and plasmid characterization}

Plasmids were transferred by broth mating to $E$. coli rifampicin-resistant K-12 J53 recipient strains and rep-typed using a PCR-based replicon typing (PBRT) commercial kit (Diatheva, Fano, Italy). The sizes of plasmids of native strains were determined by S1-Pulsed-field gel electrophoresis (PFGE) gels (Dierikx et al., 2010). Southern blot hybridizations were performed with $b l a_{\mathrm{CTX}-\mathrm{M}}, b l a_{\mathrm{TEM}}, b l a_{\mathrm{SHV}}$ or $b l a_{\mathrm{CMY}}$ probes, and probes corresponding to the various replicon types found, either on transconjugants when successfully obtained, or on native strains. Plasmid subtypes for IncI1 replicons were determined using the plasmid Multi-Locus Sequence Typing (pMLST; http://pubmlst.org/ plasmid/).

\subsection{Typing of the bacteria}

Phylogenetic grouping of the E. coli isolates was performed and subgrouped as previously described (Doumith et al., 2012). A PCR specific for the detection of the ST131 clone was performed on all B2 isolates (Clermont et al., 2009). PFGE was performed using the restriction enzyme XbaI. The software BioNumerics ${ }^{\mathrm{TM}}$ (Applied Maths, Sint-Martens-Latem, Belgium) was used for dendrogram construction and clustering, based on the band-based Dice similarity coefficient and the unweighted pair group method using arithmetic averages (UPGMA). Isolates were considered to belong to the same cluster when similarity coefficient was $\geq 90 \%$.

\subsection{Statistical analysis}

The 95\% confidence interval of the prevalence was calculated using exact confidence intervals for a binomial proportion.

\section{Results and discussion}

\subsection{Bacterial isolates and antibiotic susceptibility testing}

As the required precision (10\%) was reached rapidly due to the considerably high prevalence of ESBL-positive isolates, the sampling was stopped at 48 pieces of chicken meat in January 2016. Indeed, of the 48 pieces of chicken meat tested, $44(n=44 / 48,91.7 \%$; $95 \%$ confidence interval: [80.0-97.7]) were positive for ESC-resistant E. coli on selective plates. This prevalence is among the highest reported in Europe but comparable to what has been described in the Netherlands (94\% ESBL-producing E. coli out of 98 chicken breasts; (Leverstein-van Hall et al., 2011)) and Spain (93.3\% ESBL-producing E. coli out of 15 chicken meat samples; (Egea et al., 2012)). This still very high prevalence of ESC-resistant $E$. coli on chicken meat at retail in 2015/2016 is surely surprising as it suggests that the efforts made to decrease the use of antibiotics in poultry would not have a direct impact on the contamination of chicken meat by ESBLs/pAmpCs.

The vast majority of the isolates displayed an ESBL phenotype (74/ $77,96.1 \%$ ) while only three isolates presented a pAmpC phenotype (3/ 77, 3.9\%). The predominance of ESBL- over pAmpC-producers was expected since the pAmpC phenotype in European chicken meat was reported in a more limited number of cases compared to ESBLs, such as in Sweden importing contaminated chicks from the UK or in the UK importing raw meat from South America where AmpCs are highly prevalent (Dhanji et al., 2010; Egervarn et al., 2014; Warren et al., 
Table 1

Characteristics of the $77 \mathrm{ESBL} / \mathrm{pAmpC}$-producing E. coli isolates.

\begin{tabular}{|c|c|c|c|c|c|c|c|c|c|}
\hline \multirow[t]{3}{*}{ ESBL gene } & \multirow[t]{3}{*}{$\mathrm{Nr}$ of isolates } & \multirow[t]{3}{*}{ Phylogroup } & \multicolumn{7}{|c|}{ Plasmid types/subtypes } \\
\hline & & & \multicolumn{3}{|c|}{ IncI1 } & \multirow[t]{2}{*}{ IncFII } & \multirow[t]{2}{*}{ IncA/C } & \multirow[t]{2}{*}{ IncK } & \multirow[t]{2}{*}{ IncX1 } \\
\hline & & & ST3 & ST26 & ST95 & & & & \\
\hline \multirow[t]{4}{*}{$b l a_{\mathrm{CTX}-\mathrm{M}-1}$} & 68 & A & 20 & - & - & 2 & - & - & - \\
\hline & & B1 & 19 & - & - & 1 & - & - & - \\
\hline & & B2 & 2 & - & - & - & - & - & - \\
\hline & & $\mathrm{D}$ & 24 & - & - & - & - & - & - \\
\hline \multirow[t]{3}{*}{$b l a_{\mathrm{CMY}-2}$} & 3 & A & - & - & - & - & 1 & - & - \\
\hline & & B2 & - & - & - & - & 1 & - & - \\
\hline & & $\mathrm{D}$ & - & - & - & - & - & 1 & - \\
\hline$b l a_{\mathrm{SHV}-12}$ & 1 & B1 & - & - & 1 & - & - & - & - \\
\hline \multirow[t]{3}{*}{$b l a_{\mathrm{TEM}-52}$} & 5 & A & - & 2 & - & - & - & - & - \\
\hline & & B1 & - & - & - & - & - & - & 2 \\
\hline & & $\mathrm{D}$ & - & - & - & - & - & - & 1 \\
\hline
\end{tabular}

2008). Most isolates displayed additional resistances to sulfonamides (65/77, 84.4\%), tetracyclines $(58 / 77,75.3 \%)$, trimethoprim $(40 / 77$, $51.9 \%$ ), quinolones (32/77, 41.6\%), aminoglycosides (23/77, 29.9\%), phenicols $(11 / 77,14.3 \%)$ and fluoroquinolones $(16 / 77,20.8 \%)$, but all of them remained susceptible to ertapenem and colistin. The highest prevalence of resistance was for those antibiotics mostly used to treat poultry by the oral route in France, i. e. tetracyclines, aminoglycosides and sulfonamides. Of note, colistin is also widely used to treat animals but no resistant isolate was detected here. The dissemination of ESCresistant $E$. coli might thus also be mediated by co-selection through the use of old but still widely-used veterinary-licensed molecules.

The two protocols used (see Materials and methods section) allowed recovering a total of 93 isolates. Based on the PFGE profiles and molecular characteristics of these isolates, 26 meat samples (59.1\%) presented at least two different $E$. coli clones and a final total of 77 nonclonal isolates were kept for further studies (Table 1). There was no significant difference in the ESBL/pAmpC prevalence in meat of different commercial brands. In $15 \mathrm{ESBL} / \mathrm{pAmpC}$-positive meat samples (15/44, 34\%), only one protocol was successful (Table S1). However, both were equally effective since protocol B allowed the detection of 7 positive meat samples that were negative with protocol $\mathrm{A}$, whereas protocol A alone allowed the detection of 8 positive meat samples that were negative with protocol $\mathrm{B}$. The reasons of this divergence remain unknown but may be due to a particularly low bacterial load in these samples, which lowers the chances of finding ESBL-positive E. coli. In any case, this highlights the recurrent difficulty of comparing studies and being accurate in the detection of resistant sub-populations, even using an enrichment step (Randall et al., 2016).

\subsection{ESBL/pAmpC genes and characterization of the ESBL/pAmpC genes- carrying plasmids}

Among the ESBL isolates, the $b l a_{\mathrm{CTX}-\mathrm{M}-1}$ gene was by far the most prevalent $(68 / 74,91.9 \%)$, even though bla $_{\mathrm{TEM}-52}(5 / 74,6.8 \%)$ and bla $_{\mathrm{SHV}-12}(1 / 74,1.4 \%)$ genes were also sporadically found (Table S1). Almost all bla $_{\text {CTX-M-1 }}$ genes $(65 / 68,95.6 \%)$ were harbored by IncI1/ST3 plasmids that were mostly conjugative and whose size ranged from 97 to $120 \mathrm{~kb}$ (with the exception of one $195 \mathrm{~kb}$-sized plasmid). The remaining bla $a_{\text {CTX-M-1 }}$ genes $(3 / 68,4.4 \%)$ were carried by $120-150 \mathrm{~kb}$ IncFII plasmids. Three out of the five $b l a_{\mathrm{TEM}-52}$ genes were harbored by small ( $\sim 45 \mathrm{~kb})$ IncX1 plasmids, whereas the two last ones were carried by $\sim 100 \mathrm{~kb}$ IncI1/ST36 plasmids. The $b l a_{\mathrm{SHV}-12}$ gene was harbored by a $130 \mathrm{~kb}$ IncI1/ST95 plasmid. Only three isolates presented a pAmpC phenotype due to the presence of the $b l a_{\mathrm{CMY}-2}$ gene $(3 / 77,3.9 \%)$. Two bla $_{\mathrm{CMY}-2}$ genes were harbored by $165 \mathrm{~kb}$ IncA/C plasmids, and the third one by a $90 \mathrm{~kb}$ IncK plasmid.

The higher prevalence of the $b l a_{\mathrm{CTX}-\mathrm{M}-1}$ gene was expected and our results are in line with other European studies showing a high prevalence of $b l a_{\mathrm{CTX}-\mathrm{M}-1}$ in the retail poultry meat (Leverstein-van Hall et al., 2011; Mnif et al., 2012; Overdevest et al., 2011; Zurfluh et al., 2014). The presence of this gene was also reported in French poultry and French broiler meat imported to Denmark (Bergenholtz et al., 2009; Girlich et al., 2007). Finally, the bla $a_{\mathrm{CTX}-\mathrm{M}-1}$ gene has mainly disseminated on plasmids, and IncI1/ST3 has been repeatedly identified as the most prevalent vehicle. IncI1/ST3 was frequently reported in Europe, but not only. Indeed, bla $_{\mathrm{CTX} \text {-M-1 }}$ IncI1/ST3 plasmids seem to have a peculiar animal tropism with numerous reports in both pets and food-producing animals (Accogli et al., 2013; Bergenholtz et al., 2009; Grami et al., 2013; Haenni et al., 2014; Rodrigues et al., 2013).

In addition to $b l a_{\mathrm{CTX}-\mathrm{M}-1}, b l a_{\mathrm{TEM}-52}$ and $b l a_{\mathrm{SHV}-12}$ were also recurrently reported to confer the ESBL phenotype in European poultry isolates (Leverstein-van Hall et al., 2011). The bla $a_{\mathrm{TEM}-52}$ gene has also been reported in diarrheic veal calves in France, although at a very low frequency (Haenni et al., 2012). Interestingly, two $E$. coli from the same supplier A displayed a $b l a_{\text {TEM-52 }}$ gene that was carried by an IncI1/ST36 plasmid, thus confirming this frequent gene-plasmid combination. In parallel, IncX1 plasmids were also proved to spread $b l a_{\text {TEM-52 }}$ here, and those plasmids were interestingly found in $E$. coli isolates recovered from the same supplier D. Moreover, two of them were isolated from $E$. coli isolates found on the same meat sample (Table S1). This may suggest the presence of an IncX1-carrying contaminant in the supplier D's production processes, and an IncI1 plasmid in the supplier A's process, possibly at the growing, slaughtering or partitioning stages of the animals.

\subsection{Typing of the isolates}

All isolates were non-clonally related, as revealed by their different PFGE profiles (Fig. 1). This clearly indicates that the bla $a_{\mathrm{CTX}-\mathrm{M}-1}$ gene has disseminated horizontally and not through clonal spread. Only five $E$. coli isolates of phylogroup D, which all presented a bla $a_{\mathrm{CTX}-\mathrm{M}-1}$-carrying IncI1 plasmid of $110 \mathrm{~kb}$, were collected from five different meat samples that were sold by two unrelated suppliers and clustered with $94.5 \%$ of similarity.

Globally, 27 (35.0\%) E. coli belonged to the phylogroup D, 25 (32.5\%) to A, $22(28.6 \%)$ to B1, and $3(3.9 \%)$ to B2. None of the B2 isolates belonged to the widespread ST131 human clone, as proved by the absence of ST131-specific amplification using the previously described PCR (Clermont et al., 2009). Recent studies showed slight variations among the prevalence of phylogroups A, B1 and D in E. coli isolates from poultry settings, but B2 was generally reported at the lowest frequency (Egea et al., 2012; Huijbers et al., 2014; Kluytmans et al., 2013). 


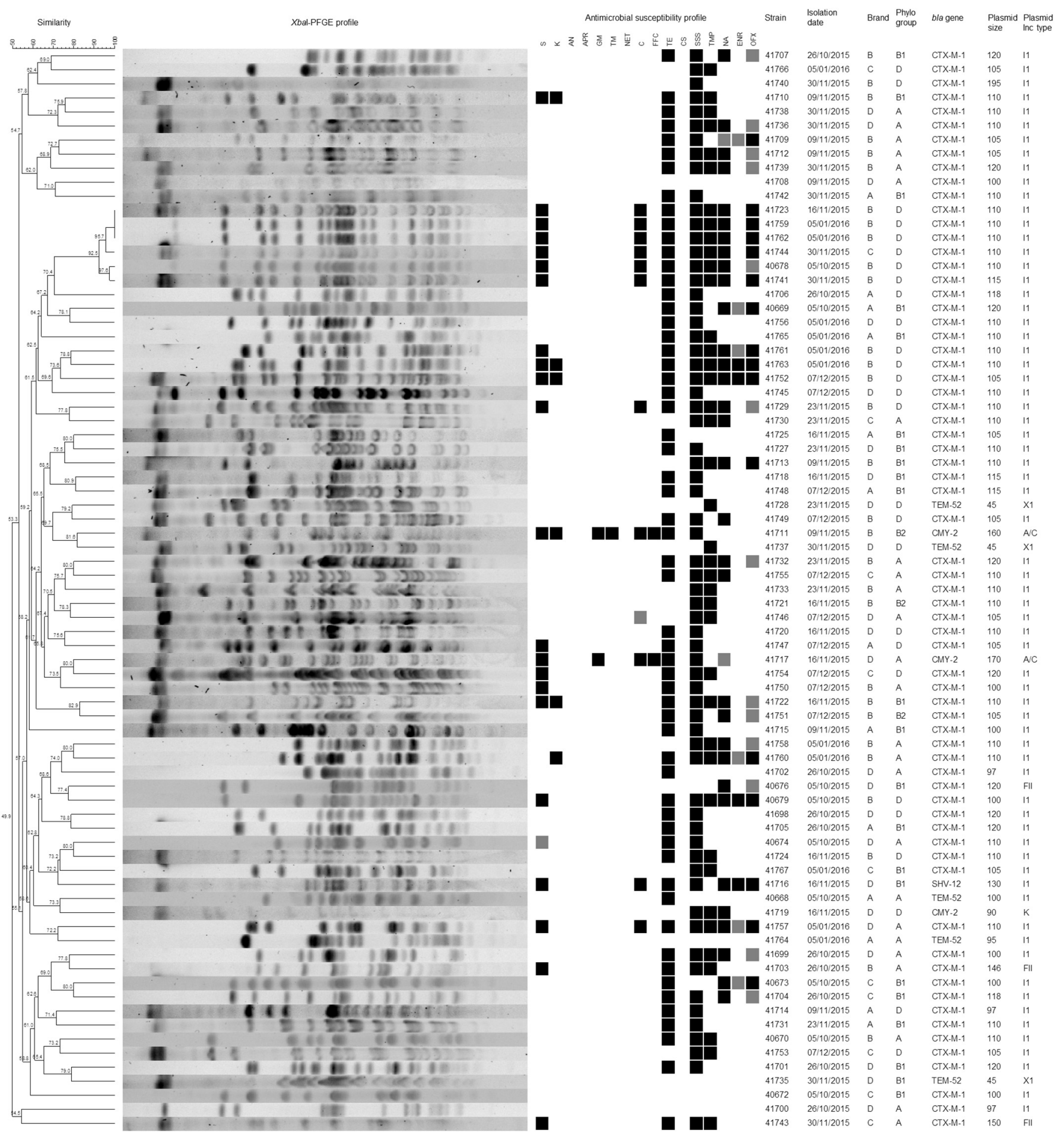

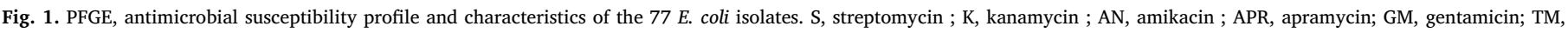


ofloxacin.

\section{Conclusions}

This study investigated the prevalence and molecular features of ESBL/pAmpC producers in retail chicken meat in France in a context of a major decrease of the use of all antimicrobials - including the off-label use of ceftiofur - in poultry in this country, in accordance to the national strategic action plan set up late 2011 in the animal sector. The importance of this study relies on the surprising discrepancy observed between the reduced use of ESC and other antibiotics globally and the still considerable ESBL/pAmpC prevalence in retail chicken meat.

These findings raise several questions as follows. It may be argued that the poultry meat samples originate from living chicken - or be themselves - introduced in France from countries with less responsible practices towards antibiotic use (Egervarn et al., 2014; Warren et al., 2008). However, imported chicken or chicken meat cannot be blamed since all poultry producers indicated a French origin of the animals. Moreover, in France, imported chicken meat is never sold raw but always processed with other food products. Also, the animal or human 
origin of the ESBL producers may be questioned, since chicken meat contamination with ESC-resistant bacteria may either reflect an antibiotic use in animals or result from human transfers through food handling and processing. However, our data rather suggest an animal origin since IncI1/ST3 plasmids carrying $b l a_{\mathrm{CTX}-\mathrm{M}-1}$ have been massively reported in animals and only sporadically in humans (Madec et al., 2015). Also, none of the E. coli isolates were of the B2 phylogroup, which includes most ESBL genes and clones in humans (and mainly the CTX-M-15-producing ST131 clone), but rarely bla $_{\text {CTX-M-1. }}$.

In all, these data make us believe that, despite a probable limited exposure of poultry to ESC since 2011/2012 in France, ESBL/pAmpC producers are still present at a high frequency in the sub-dominant gut flora of the animals in 2015/2016. Similar observations were made in human beings after exposure to antibiotics (Carlet, 2012). This is also in line with the current high ESBL/pAmpC prevalence in poultry in Europe when measured using selective media. These results question the routes of dissemination of these bacteria through the poultry pyramid and along the meat processing chain. The fact is surprising as the very short period of chicken production ( $<2$ months) and the all-in all-out production system should have favored a rapid elimination of ESBL/ pAmpC producers from poultry farms after stopping using ESCs. It is also alarming considering the potential transmission of ESBL/pAmpC producers to humans via the food chain, which may not be sufficiently counteracted by the current measures in place in the animal sector in Europe.

Supplementary data to this article can be found online at http://dx. doi.org/10.1016/j.ijfoodmicro.2017.07.005.

\section{Acknowledgements}

This work was supported by the French Agency for Food, Environmental and Occupational Health and Safety (ANSES). We thank Emilie Gay for her help with the sampling design and statistical analyses. Tiago Casella was supported by the Foundation Coordination for the Improvement of Higher Education Personnel (CAPES) - scholar 99999.009932/2014-03, of the Ministry of Education, Brazil.

\section{References}

Accogli, M., Fortini, D., Giufre, M., Graziani, C., Dolejska, M., Carattoli, A., Cerquetti, M., 2013. IncI1 plasmids associated with the spread of CMY-2, CTX-M-1 and SHV-12 in Escherichia coli of animal and human origin. Clin. Microbiol. Infect. 19, E238-E240.

ANSES, 2014. Sales Survey of Veterinary Medicinal Products Containing Antimicrobials in France - 2013.

Bergenholtz, R.D., Jorgensen, M.S., Hansen, L.H., Jensen, L.B., Hasman, H., 2009. Characterization of genetic determinants of extended-spectrum cephalosporinases (ESCs) in Escherichia coli isolates from Danish and imported poultry meat. J. Antimicrob. Chemother. 64, 207-209.

Borjesson, S., Egervarn, M., Lindblad, M., Englund, S., 2013. Frequent occurrence of extended-spectrum beta-lactamase- and transferable ampc beta-lactamase-producing Escherichia coli on domestic chicken meat in Sweden. Appl. Environ. Microbiol. 79, 2463-2466.

Carlet, J., 2012. The gut is the epicentre of antibiotic resistance. Antimicrob Resist Infect Control 1, 39.

Clermont, O., Dhanji, H., Upton, M., Gibreel, T., Fox, A., Boyd, D., Mulvey, M.R., Nordmann, P., Ruppe, E., Sarthou, J.L., Frank, T., Vimont, S., Arlet, G., Branger, C., Woodford, N., Denamur, E., 2009. Rapid detection of the O25b-ST131 clone of Escherichia coli encompassing the CTX-M-15-producing strains. J. Antimicrob. Chemother. 64, 274-277.

Dallenne, C., Da Costa, A., Decre, D., Favier, C., Arlet, G., 2010. Development of a set of multiplex PCR assays for the detection of genes encoding important beta-lactamases in Enterobacteriaceae. J. Antimicrob. Chemother. 65, 490-495.

Dhanji, H., Murphy, N.M., Doumith, M., Durmus, S., Lee, S.S., Hope, R., Woodford, N., Livermore, D.M., 2010. Cephalosporin resistance mechanisms in Escherichia coli isolated from raw chicken imported into the UK. J. Antimicrob. Chemother. 65, 2534-2537.

Dierikx, C., van Essen-Zandbergen, A., Veldman, K., Smith, H., Mevius, D., 2010. Increased detection of extended spectrum beta-lactamase producing Salmonella enterica and Escherichia coli isolates from poultry. Vet. Microbiol. 145, 273-278.

Dierikx, C.M., van der Goot, J.A., Smith, H.E., Kant, A., Mevius, D.J., 2013. Presence of ESBL/AmpC-producing Escherichia coli in the broiler production pyramid: a descriptive study. PLoS One 8, e79005.
Doumith, M., Day, M.J., Hope, R., Wain, J., Woodford, N., 2012. Improved multiplex PCR strategy for rapid assignment of the four major Escherichia coli phylogenetic groups. J. Clin. Microbiol. 50, 3108-3110.

Dutil, L., Irwin, R., Finley, R., Ng, L.K., Avery, B., Boerlin, P., Bourgault, A.M., Cole, L., Daignault, D., Desruisseau, A., Demczuk, W., Hoang, L., Horsman, G.B., Ismail, J., Jamieson, F., Maki, A., Pacagnella, A., Pillai, D.R., 2010. Ceftiofur resistance in Salmonella enterica serovar Heidelberg from chicken meat and humans, Canada. Emerg. Infect. Dis. 16, 48-54.

Efsa Panel on Biological Hazards, E.P.o.C.i.t.F.C., Health, E.P.o.A., Welfare, 2012. Scientific Opinion on the public health hazards to be covered by inspection of meat (poultry). EFSA J. 10, 2741-n/a.

Egea, P., Lopez-Cerero, L., Torres, E., Gomez-Sanchez Mdel, C., Serrano, L., Navarro Sanchez-Ortiz, M.D., Rodriguez-Bano, J., Pascual, A., 2012. Increased raw poultry meat colonization by extended spectrum beta-lactamase-producing Escherichia coli in the south of Spain. Int. J. Food Microbiol. 159, 69-73.

Egervarn, M., Borjesson, S., Byfors, S., Finn, M., Kaipe, C., Englund, S., Lindblad, M., 2014. Escherichia coli with extended-spectrum beta-lactamases or transferable AmpC beta-lactamases and Salmonella on meat imported into Sweden. Int. J. Food Microbiol. 171, 8-14.

EMA, 2017. EMA and EFSA Joint Scientific Opinion on measures to reduce the need to use antimicrobial agents in animal husbandry in the European Union, and the resulting impacts on food safety (RONAFA). EFSA J. 15, e04666-n/a.

FAO, 2015. FAOSTAT: Food Balance/Food Supply - Livestock and Fish Primary Equivalent [WWW Document]. Food Agric. Organ. United Nations - Stat. Div. http:// faostat3.fao.org/browse/FB/CL/E.

Girlich, D., Poirel, L., Carattoli, A., Kempf, I., Lartigue, M.F., Bertini, A., Nordmann, P., 2007. Extended-spectrum beta-lactamase CTX-M-1 in Escherichia coli isolates from healthy poultry in France. Appl. Environ. Microbiol. 73, 4681-4685.

Grami, R., Mansour, W., Dahmen, S., Mehri, W., Haenni, M., Aouni, M., Madec, J.Y.,


J. Antimicrob. Chemother. 68, 2950-2952.

Haenni, M., Saras, E., Metayer, V., Doublet, B., Cloeckaert, A., Madec, J.Y., 2012. Spread of the bla $a_{\mathrm{TEM}-52}$ gene is mainly ensured by IncI1/ST36 plasmids in Escherichia coli isolated from cattle in France. J. Antimicrob. Chemother. 67, 2774-2776.

Haenni, M., Saras, E., Metayer, V., Medaille, C., Madec, J.Y., 2014. High prevalence of $b_{l a} a_{\mathrm{CTX}-\mathrm{M}-1} / \mathrm{IncI} 1 / \mathrm{ST} 3$ and bla $_{\mathrm{CMY}-2} / \mathrm{IncI1} / \mathrm{ST} 2$ plasmids in healthy urban dogs in France. Antimicrob. Agents Chemother. 58, 5358-5362.

Huijbers, P.M., Graat, E.A., Haenen, A.P., van Santen, M.G., van Essen-Zandbergen, A., Mevius, D.J., van Duijkeren, E., van Hoek, A.H., 2014. Extended-spectrum and AmpC beta-lactamase-producing Escherichia coli in broilers and people living and/or working on broiler farms: prevalence, risk factors and molecular characteristics. J. Antimicrob. Chemother. 69, 2669-2675.

Kluytmans, J.A., Overdevest, I.T., Willemsen, I., Kluytmans-van den Bergh, M.F., van der Zwaluw, K., Heck, M., Rijnsburger, M., Vandenbroucke-Grauls, C.M., Savelkoul, P.H., Johnston, B.D., Gordon, D., Johnson, J.R., 2013. Extended-spectrum beta-lactamaseproducing Escherichia coli from retail chicken meat and humans: comparison of strains, plasmids, resistance genes, and virulence factors. Clin. Infect. Dis. 56, 478-487.

Leverstein-van Hall, M.A., Dierikx, C.M., Cohen Stuart, J., Voets, G.M., van den Munckhof, M.P., van Essen-Zandbergen, A., Platteel, T., Fluit, A.C., van de SandeBruinsma, N., Scharinga, J., Bonten, M.J., Mevius, D.J., 2011. Dutch patients, retail chicken meat and poultry share the same ESBL genes, plasmids and strains. Clin. Microbiol. Infect. 17, 873-880.

Madec, J.Y., Haenni, M., Metayer, V., Saras, E., Nicolas-Chanoine, M.H., 2015. High prevalence of the animal-associated $b l a_{\text {CTX-M-1 }}$ IncI1/ST3 plasmid in human Escherichia coli isolates. Antimicrob. Agents Chemother. 59, 5860.

Mnif, B., Ktari, S., Rhimi, F.M., Hammami, A., 2012. Extensive dissemination of CTX-M-1and CMY-2-producing Escherichia coli in poultry farms in Tunisia. Lett. Appl. Microbiol. 55, 407-413.

Overdevest, I., Willemsen, I., Rijnsburger, M., Eustace, A., Xu, L., Hawkey, P., Heck, M., Savelkoul, P., Vandenbroucke-Grauls, C., van der Zwaluw, K., Huijsdens, X., Kluytmans, J., 2011. Extended-spectrum beta-lactamase genes of Escherichia coli in chicken meat and humans, The Netherlands. Emerg. Infect. Dis. 17, 1216-1222.

Randall, L.P., Lodge, M.P., Elviss, N.C., Lemma, F.L., Hopkins, K.L., Teale, C.J., Woodford, N., 2016. Evaluation of meat, fruit and vegetables from retail stores in five United Kingdom regions as sources of extended-spectrum beta-lactamase (ESBL)-producing and carbapenem-resistant Escherichia coli. Int. J. Food Microbiol. 241, 283-290.

Rodrigues, C., Machado, E., Peixe, L., Novais, A., 2013. IncI1/ST3 and IncN/ST1 plasmids drive the spread of blaTEM-52 and blaCTX-M-1/-32 in diverse Escherichia coli clones from different piggeries. J. Antimicrob. Chemother (in press).

Shibata, N., Kurokawa, H., Doi, Y., Yagi, T., Yamane, K., Wachino, J.-I., Suzuki, S., Kimura, K., Ishikawa, S., Kato, H., Ozawa, Y., Shibayama, K., Kai, K., Konda, T., Arakawa, Y., 2006. PCR classification of CTX-M-type beta-lactamase genes identified in clinically isolated gram-negative bacilli in Japan. Antimicrob. Agents Chemother. 50, 791-795.

Warren, R.E., Ensor, V.M., O'Neill, P., Butler, V., Taylor, J., Nye, K., Harvey, M., Livermore, D.M., Woodford, N., Hawkey, P.M., 2008. Imported chicken meat as a potential source of quinolone-resistant Escherichia coli producing extended-spectrum beta-lactamases in the UK. J. Antimicrob. Chemother. 61, 504-508.

Zurfluh, K., Wang, J., Klumpp, J., Nuesch-Inderbinen, M., Fanning, S., Stephan, R., 2014. Vertical transmission of highly similar $b l a_{\mathrm{CTX}-\mathrm{M}-1}$-harboring IncI1 plasmids in Escherichia coli with different MLST types in the poultry production pyramid. Front. Microbiol. 5, 519. 